\title{
Current Management of the Complications of Cirrhosis and Portal Hypertension: Variceal Hemorrhage, Ascites, and Spontaneous Bacterial Peritonitis
}

\author{
Guadalupe Garcia-Tsao a, b
}

Section of Digestive Diseases, a Yale University School of Medicine, New Haven, C.T., and bVA-Connecticut Healthcare System, West Haven, C.T., USA

\section{Key Words}

Cirrhosis · Infection · Multidrug resistant organisms .

Antibiotic prophylaxis

\begin{abstract}
Cirrhosis is not a single entity but represents a disease progression across different prognostic stages, with the compensated and decompensated stages being the most important. Variceal hemorrhage $(\mathrm{VH})$ and ascites are complications of cirrhosis that denote the presence of a decompensated stage. Spontaneous bacterial peritonitis (SBP) is a common bacterial infection unique to patients with cirrhosis that can precipitate the development of recurrent VH and hepatorenal syndrome (HRS), complications that denote the presence of a 'further decompensated' stage of cirrhosis. Main current issues in the management of $\mathrm{VH}$ include identification of different prognostic stages that allow for individualized patient care. Management of $\mathrm{VH}$ cannot be performed in an isolated manner, and the presence of other complications of cirrhosis (ascites, encephalopathy) should be taken into account both in the management and in the design of clinical trials. Because management of ascites per se has not resulted in significant changes in mortality, main management issues consist of preventing further decompensating events by preventing factors that will lead to worsening vasodilatation and hemodynamic status (infections, vasodilators), prevent-
\end{abstract}

ing volume depletion (overdiuresis, Gl hemorrhage) and preventing structural kidney injury (nephrotoxins). Prophylaxis of bacterial infections such as SBP currently consists of the administration of antibiotics. By preventing infections, there is evidence that recurrent $\mathrm{VH}$ and HRS can also be prevented. However, response to recommended empirical antibiotics in patients with suspected infection, such as SBP, is currently significantly lower than in the past because of an increase in infections secondary to multidrug resistant (MDR) organisms. One of the main predictors of the development of MDR organisms is antibiotic prophylaxis and unnecessary and prolonged use of antibiotics in hospital. Therefore, appropriate antibiotics should be used in patients with a high suspicion of infection, and antibiotic prophylaxis should be restricted to patients with the highest risk of infection.

(c) 2016 S. Karger AG, Basel

Numerous prognostic studies over the years have demonstrated that cirrhosis is not a single entity. At a minimum, cirrhosis should be divided in 2 stages depending on the presence or absence of clinically evident decompensating events (specifically ascites, variceal hemorrhage (VH), hepatic encephalopathy and jaundice); 1-year survival is around $95 \%$ in compensated patients, that is, those without any clinical decompensating events, while in decompensated patients, it is around 61\% [1]. Analysis of

\section{KARGER}

E-Mail karger@karger.com

www.karger.com/ddi
(C) 2016 S. Karger AG, Basel

0257-2753/16/0344-0382\$39.50/0
Guadalupe Garcia-Tsao, MD

Digestive Diseases Section

Yale University/VA-CT Heathcare System

333 Cedar Street - 1080 LMP, New Haven, CT 06460 (USA)

E-Mail guadalupe.gacia-tsao@yale.edu 
individual patient data from 2 prospective Italian cohort studies that included over 1,600 patients demonstrated a median survival of $>12$ years in patients with compensated cirrhosis, while patients with decompensated cirrhosis had a median survival of 1.8 years [1]. In fact, when cirrhosis is taken as a whole, decompensation is the strongest predictor of death (Ripoll, 2015 3287/id).

\section{Variceal Hemorrhage}

One of the complications that define decompensation is $\mathrm{VH}$, a complication that results directly from portal hypertension.

In patients with $\mathrm{VH}$, the goal of therapy is to control acute hemorrhage and to prevent its early recurrence (within 5 days) and death. Mortality from VH has decreased over the years because of implementation of therapies that have had an impact on survival, specifically the use of antibiotic prophylaxis, the use of ligation (in lieu of sclerotherapy) and the use of safe vasoactive drugs - therapies that represent the current standard of care [3]. Since then, a couple of additional strategies have led to further improvements in survival.

One of them is a restrictive transfusion strategy. Packed red blood cell transfusion should be done conservatively for a target hemoglobin level between 7-9 $\mathrm{g} / \mathrm{dl}$ because a more liberal transfusion strategy in patients with cirrhosis (i.e. transfusing for a target hemoglobin of 9-11 g/dl) has been shown in a randomized controlled trial (RCT) to be associated with increased mortality and a significant increase in hepatic venous pressure gradient [4].

The other is the use of 'early', preemptive, placement of transjugular intrahepatic portosystemic shunt (TIPS) in patients at a high risk of failing standard therapy, mainly consisting of patients in Child C class (excluding those with a score of 14 and 15). In these patients, once vasoactive drugs have been initiated and EVL performed, placement of TIPS (ideally within $24 \mathrm{~h}$ of admission) must be considered as it has been shown to reduce failure of therapy and to improve survival $[5,6]$. Notably, these patients constitute $<20 \%$ of those admitted for $\mathrm{VH}$.

$\mathrm{VH}$ can occur either as an isolated complication or in conjunction with other complications (ascites and/or encephalopathy), and this, as expected, has different prognostic implications. Five-year mortality in patients who present with VH without other complication is $20 \%$ while mortality in patients with $\mathrm{VH}$ and another complication can be as high as $88 \%$ [7]. Therefore, in patients whose only complication is VH (and this has been controlled), the objective of therapy is not only to prevent recurrent hemorrhage but also to prevent the development of these other complications [8].

Given the high rebleeding risk in patients who have recovered from an episode of $\mathrm{VH}(60 \%$ in the first year with a mortality of up to $33 \%$ ), prevention of rebleeding is an essential part of their management.

Patients who had a TIPS placed during the acute episode do not require specific therapy for portal hypertension or for varices but should be referred for transplant evaluation. TIPS patency should be checked with Doppler ultrasound every 6 months. First-line therapy for all other patients (the majority) is the combination of nonselective beta-blockers (NSBB, propranolol or nadolol) + EVL. A recent meta-analysis comparing combination therapy to monotherapy with EVL or drug therapy has demonstrated that, when compared to EVL, combination therapy is significantly more effective in preventing all-source GI hemorrhage. However, combination therapy is only marginally more effective than drug therapy (NSBB \pm nitrates) alone with a tendency for an increased survival with drugs alone [9]. This suggests that pharmacological therapy is the cornerstone of combination therapy and that EVL should not be used as sole therapy in the prevention of recurrent hemorrhage. In patients who cannot tolerate NSBB (see below), TIPS should be considered to prevent rebleeding. TIPS (using the smaller diameter $8 \mathrm{~mm}$ stents) has been recently compared to hepatic venous pressure gradient-guided therapy in the prevention of rebleeding in patients with Child A or B cirrhosis showing that TIPS prevented rebleeding more effectively than drugs, but without a survival benefit, and was associated with more adverse events (mainly encephalopathy) [10].

\section{Ascites}

Ascites is the most common decompensating event in cirrhosis and the one that carries the highest mortality. Therapies for ascites are those directed at increasing sodium excretion via the use of diuretics and/or removing fluid via large-volume paracentesis. Both these therapies act downstream of the main pathophysiological mechanisms that lead to ascites formation and none of them have been shown to improve survival. The only therapy that appears to improve survival in patients with recurrent/refractory ascites is the use of TIPS, particularly in those with a MELD $<15$ [11]. 
The goal of therapy in patients with ascites is to prevent a worsening of the pathophysiological mechanisms that lead to the formation of ascites and thereby prevent 'further' decompensation (hyponatremia, acute kidney injury (AKI)). One of the main factors that contribute to the development of hyponatremia and hepatorenal syndrome (HRS) in cirrhosis is vasodilatation of both the splanchnic and systemic circulations [12]. This vasodilatation occurs after portal hypertension has led to the formation of portosystemic collaterals and leads to decreased effective arterial blood volume and activation of various vasoconstrictor and anti-natriuretic neurohumoral systems (the reninangiotensin-aldosterone system and sympathetic nervous system), leading to renal sodium and water retention and to an increase in intravascular volume, which in turn leads to a hyperdynamic circulatory state (low systemic vascular resistance, high cardiac output).

In advanced stages of cirrhosis, progressive vasodilatation leads not only to avid sodium retention (with formation of ascites that is now refractory to diuretics) but also to the non-osmotic release of antidiuretic hormone or arginine vasopressin (AVP). When activated by AVP, $V_{2}$ receptors in the kidney tubule enable translocation of selective water channels called aquaporins from the cytosol to the luminal plasma membrane of the collecting ducts, increasing water permeability. This increase in water reabsorption exceeds that of sodium retention and leads to dilutional hyponatremia. $\mathrm{V}_{2}$ receptor antagonism improves hyponatremia as long as patients are on these drugs, and in patients with cirrhosis and severe hyponatremia $(<127 \mathrm{mEq} / \mathrm{l})$, the effect seems to be only transient [13].

Progressive vasodilatation also leads to further activation of vasoconstrictive systems (mainly renin and angiotensin), resulting in renal vasoconstriction and decreased renal blood flow. In addition, a relative decrease in cardiac output in this high-output cardiac state (or cirrhotic cardiomyopathy) may further contribute to decreased renal blood flow [14]. This decrease in renal blood flow leads to a decreased glomerular filtration rate and a prerenal type of kidney injury, that is, HRS.

Therefore, in patients with ascites, particularly in those with refractory ascites, factors that will worsen vasodilatation should be avoided/prevented. Therefore, vasodilators (angiotensin receptor blockers, ACE inhibitors, carvedilol) should not be used in these patients, particularly if associated with a decrease (even minimal) in arterial blood pressure. In this context, the use of NSBB could further decrease cardiac output and destabilize patients who are in this fragile hemodynamic state theoretically leading to further decompensation, AKI and death. Sersté et al. [15] showed, in a prospective non-matched prospective study, that propranolol use (at the time of diagnosis of refractory ascites) led to a higher mortality than patients not on propranolol. This has led practitioners to discontinue NSBB in patients with cirrhosis (even in those with diuretic-responsive ascites). This is particularly unjustifiable in light of 2 more recent larger studies that have shown a greater survival with NSBB or no differences in survival, respectively. In the first of these studies, a propensity score-matched study in a cohort of patients with cirrhosis on the transplant wait list showed a survival advantage of patients with refractory ascites on NSBB compared to those not on NSBB and, interestingly, showed that the use of carvedilol (a NSBB with vasodilatory properties that has been shown to lead to a larger decrease in blood pressure compared to propranolol [16]) had an intermediate survival between patients with propranolol (lowest mortality) and those not on NSBB (highest mortality) [17]. The second study, a post-hoc analysis of data from 3 RCTs about $V_{2}$ receptor antagonists in the treatment of ascites, showed no differences in mortality between those on and off NSBB, even when adjusted by MELD, mean arterial pressure, dose of beta-blockers or study group (satavaptan vs. placebo) [18].

Given the benefit of NSBB, particularly in the prevention of recurrent $\mathrm{VH}$, the Baveno VI consensus conference recommended that, until further evidence is available, NSBB should be used cautiously in patients with refractory ascites and dose reduced/discontinued in the presence of a systolic blood pressure $<90 \mathrm{~mm} \mathrm{Hg}$, serum sodium $<130 \mathrm{mEq} / 1$ or development of AKI [8].

In the setting of prevention of recurrent $\mathrm{VH}$, carvedilol has only been compared to EVL alone [19] or to NSBB + ISMN [20] but has not been compared to standard of care with the combination of NSBB + EVL. Therefore, carvedilol is not recommended in this setting [8]. Furthermore, since carvedilol leads to a larger decrease in blood pressure [16], it should be avoided in patients with refractory ascites even in the setting of primary prophylaxis of $\mathrm{VH}$.

\section{Spontaneous Bacterial Peritonitis}

Bacterial infections are a common complication in hospitalized patients with cirrhosis and are the most common precipitant of acute-on-chronic liver failure (AOCLF) [21] - entity in which worsening vasodilatation is a major pathogenic mechanism. It is estimated that approximately 
a third of patients are admitted with an infection or develop it during admission. This is in contrast to the rate of infections in the hospitalized non-cirrhotic population which is $5-7 \%$. Infections are associated with a high mortality, with a 4-fold increase in in-hospital mortality [22].

The most common bacterial infections in cirrhosis are the so-called 'spontaneous' infections (accounting for a third), that is, spontaneous bacterial peritonitis (SBP), spontaneous bacterial empyema (SBE) and spontaneous bacteremia, followed by urinary tract infection and pneumonia [23]. Any of these infections can lead to further decompensation with development of AKI, jaundice, coagulopathy and/or encephalopathy that result from worsening vasodilatation and a systemic inflammatory state. Failure of more than 2 organs (liver, kidney, brain, coagulation system, circulatory system and/or lungs) defines AOCLF, carrying a high 28-day mortality ( 30\%) [21]. Specifically, for infection-induced AOCLF, failure of 2 organs has been shown to be associated with a 30-day mortality of $\sim 50 \%$ [24].

Prompt and appropriate empirical antibiotic treatment of infection and early resuscitation of patients with severe sepsis or septic shock are essential in determining the patient outcome. The majority ( $>80 \%$ ) of community-acquired infections resolves with empirical antibiotic therapy [23]. For spontaneous infections (SBP, SBE), the recommended empirical antibiotic is a third-generation cephalosporin, preferably intravenous cefotaxime $2 \mathrm{~g}$ administered every $12 \mathrm{~h} \mathrm{[25].}$

However, the prevalence of infections due to multidrug resistant (MDR) bacteria has been increasing in an alarming fashion. In a study performed in Spain, the prevalence doubled from $<10 \%$ between 1998 and 2000 to $23 \%$ between 2010 and 2011 [26]. In a tertiary US series, the prevalence of infections due to MDR organism was $47 \%$ [27]. Infections due to MDR organisms are responsible for increasing rates of non-response to recommended empirical antibiotics and are associated with an increased risk of septic shock, AKI and death, both in the pre- and the post-transplant settings [26].

Predictors of the development of MDR infections are frequent and suboptimal antibiotic use (including antibiotic prophylaxis), nosocomial acquisition of infection and recent infection with a MDR organism [26, 27].

Antibiotic stewardship principles should be implemented in each hospital and include the prevention of antibiotic overuse (identification of early diagnostic tests to diagnose an infection), early de-escalation policies as well as shorter courses of antibiotics. Prescription of empiric antibiotics should be made according to the severity

Current Management of the Complications

of Cirrhosis and Portal Hypertension of infection (broader schemes in more severe cases), the presence or absence of risk factors for the development of MDR bacteria and the local epidemiology [28].

Even though antibiotic prophylaxis has led to a decrease in bacterial infections and, as proof of concept, has also led to a decrease in further decompensating events in cirrhosis (recurrent VH, HRS), it has also been associated with the development of infections due to MDR organisms [28]. Therefore, antibiotic prophylaxis should also be minimized and limited only to patients who are at the highest risk of developing a bacterial infection as is currently recommended in practice guidelines of both the American Association for the Study of Liver Diseases [29] and the European Association for the Study of the Liver [30]. These patients are the following:

(1) Patients with cirrhosis presenting with upper GI hemorrhage: per recent Baveno VI recommendations [8], antibiotic prophylaxis with norfloxacin $400 \mathrm{mg} / 12 \mathrm{~h}$ p.o. for 7 days should be instituted from the time of admission, with intravenous ceftriaxone at $1 \mathrm{~g} / 24 \mathrm{~h}$ for 7 days recommended in patients with advanced cirrhosis, in hospital settings with a high prevalence of quinolone-resistant bacterial infections and in patients on previous quinolone prophylaxis.

(2) Patients who have survived an episode of SBP: secondary prophylaxis with norfloxacin at $400 \mathrm{mg} /$ day p.o. is the recommended prophylaxis. Alternatively, since norfloxacin is no longer available in the US; ciprofloxacin at a dose of $500 \mathrm{mg} /$ day can be used instead. Treatment should continue until liver transplant, death or resolution of ascites or improvement in liver function to a compensated stage.

(3) Patients with ascitic fluid protein $<1.5 \mathrm{~g} / \mathrm{dl}$ along with impaired renal function (creatinine $\geq 1.2, B U N \geq 25$, or serum $\mathrm{Na} \leq 130$ ) or liver failure (child score $\geq 9$ and bilirubin $\geq 3 \mathrm{mg} / \mathrm{dl}$ ): primary prophylaxis with longterm norfloxacin $400 \mathrm{mg}$ /day p.o. or ciprofloxacin 500 $\mathrm{mg} /$ day is recommended in these patients. This is particularly relevant in patients awaiting liver transplantation for whom the development of an infection would prompt removal from the transplant list.

\section{Grant Support}

NIH P-30DK 034989.

\section{Disclosure Statement}

The author has no disclosures relevant to this article. 


\section{References}

1 D'Amico G, Garcia-Tsao G, Pagliaro L: Natural history and prognostic indicators of survival in cirrhosis: a systematic review of 118 studies. J Hepatol 2006;44:217-231.

2 Ripoll C, Bari K, Garcia-Tsao G: Serum albumin can identify patients with compensated cirrhosis with a good prognosis. J Clin Gastroenterol 2015;49:613-619.

3 Garcia-Tsao G, Sanyal AJ, Grace ND, Carey W; Practice Guidelines Committee of the American Association for the Study of Liver Diseases; Practice Parameters Committee of the American College of Gastroenterology: Prevention and management of gastroesophageal varices and variceal hemorrhage in cirrhosis. Hepatology 2007;46:922-938.

4 Villanueva C, Colomo A, Bosch A, et al: Transfusion strategies for acute upper gastrointestinal bleeding. N Engl J Med 2013;368: 11-21.

5 García-Pagán JC, Caca K, Bureau C, et al: Early use of TIPS in patients with cirrhosis and variceal bleeding. N Engl J Med 2010;362: 2370-2379.

6 Garcia-Pagán JC, Di Pascoli M, Caca K, et al: Use of early-TIPS for high-risk variceal bleeding: results of a post-RCT surveillance study. J Hepatol 2013;58:45-50.

7 D’Amico G, Pasta L, Morabito A, et al: Competing risks and prognostic stages of cirrhosis: a 25-year inception cohort study of 494 patients. Aliment Pharmacol Ther 2014;39: 1180-1193.

8 de Franchis R; Baveno VI Faculty: Expanding consensus in portal hypertension: report of the Baveno VI consensus workshop: stratifying risk and individualizing care for portal hypertension. J Hepatol 2015;63:743-752.

9 Puente A, Hernández-Gea V, Graupera I, et al: Drugs plus ligation to prevent rebleeding in cirrhosis: an updated systematic review. Liver Int 2014;34:823-833.

10 Sauerbruch T, Mengel M, Dollinger M, et al: Prevention of rebleeding from esophageal varices in patients with cirrhosis receiving small-diameter stents versus hemodynamically controlled medical therapy. Gastroenterology 2015;149:660-668.e1.
11 Salerno F, Cammà C, Enea M, Rössle M, Wong F: Transjugular intrahepatic portosystemic shunt for refractory ascites: a metaanalysis of individual patient data. Gastroenterology 2007;133:825-834.

12 Schrier RW, Arroyo V, Bernardi M, Epstein M, Henriksen JH, Rodés J: Peripheral arterial vasodilation hypothesis: a proposal for the initiation of renal sodium and water retention in cirrhosis. Hepatology 1988;8:1151-1157.

13 Cárdenas A, Ginès P, Marotta P, et al: Tolvaptan, an oral vasopressin antagonist, in the treatment of hyponatremia in cirrhosis. J Hepatol 2012;56:571-578.

14 Ruiz-del-Arbol L, Monescillo A, Arocena C, et al: Circulatory function and hepatorenal syndrome in cirrhosis. Hepatology 2005;42: 439-447.

15 Sersté T, Melot C, Francoz C, et al: Deleterious effects of beta-blockers on survival in patients with cirrhosis and refractory ascites. Hepatology 2010;52:1017-1022.

16 Sinagra E, Perricone G, D’Amico M, Tinè F, D'Amico G: Systematic review with metaanalysis: the haemodynamic effects of carvedilol compared with propranolol for portal hypertension in cirrhosis. Aliment Pharmacol Ther 2014;39:557-568.

17 Leithead JA, Rajoriya N, Tehami N, et al: Non-selective $\beta$-blockers are associated with improved survival in patients with ascites listed for liver transplantation. Gut 2015;64: 1111-1119.

18 Bossen L, Krag A, Vilstrup H, Watson H, Jepsen P: Non-selective $\beta$-blockers do not affect mortality in cirrhosis patients with ascites: post hoc analysis of three RCTs with 1198 patients. Hepatology 2015, Epub ahead of print.

19 Stanley AJ, Dickson S, Hayes PC, et al: Multicentre randomised controlled study comparing carvedilol with variceal band ligation in the prevention of variceal rebleeding. J Hepatol 2014;61:1014-1019.

20 Lo GH, Chen WC, Wang HM, Yu HC: Randomized, controlled trial of carvedilol versus nadolol plus isosorbide mononitrate for the prevention of variceal rebleeding. J Gastroenterol Hepatol 2012;27:1681-1687.
21 Moreau R, Jalan R, Gines P, et al: Acute-onchronic liver failure is a distinct syndrome that develops in patients with acute decompensation of cirrhosis. Gastroenterology 2013;144:1426-1437.e1-e9.

22 Arvaniti V, D’Amico G, Fede G, et al: Infections in patients with cirrhosis increase mortality four-fold and should be used in determining prognosis. Gastroenterology 2010; 139:1246-1256.e1-e5.

23 Fernández J, Navasa M, Gómez J, et al: Bacterial infections in cirrhosis: epidemiological changes with invasive procedures and norfloxacin prophylaxis. Hepatology 2002;35: 140-148.

24 Bajaj JS, O’Leary JG, Reddy KR, et al: Survival in infection-related acute-on-chronic liver failure is defined by extrahepatic organ failures. Hepatology 2014;60:250-256.

25 Fernández J, Gustot T: Management of bacterial infections in cirrhosis. J Hepatol 2012; 56(suppl 1):S1-S12.

26 Fernández J, Acevedo J, Castro M, et al: Prevalence and risk factors of infections by multiresistant bacteria in cirrhosis: a prospective study. Hepatology 2012;55:15511561.

27 Tandon P, Delisle A, Topal JE, Garcia-Tsao G: High prevalence of antibiotic-resistant bacterial infections among patients with cirrhosis at a US liver center. Clin Gastroenterol Hepatol 2012;10:1291-1298.

28 Fernández J, Tandon P, Mensa J, Garcia-Tsao G: Antibiotic prophylaxis in cirrhosis: good and bad. Hepatology 2015, Epub ahead of print.

29 Runyon BA; AASLD: Introduction to the revised American association for the study of liver diseases practice guideline management of adult patients with ascites due to cirrhosis 2012. Hepatology 2013;57:16511653.

30 European Association for the Study of the Liver: EASL clinical practice guidelines on the management of ascites, spontaneous bacterial peritonitis, and hepatorenal syndrome in cirrhosis. J Hepatol 2010;53:397-417. 